\section{The effects of a brace for patellofemoral osteoarthritis targeting knee pain and bone marrow lesions were overestimated or not?}

We read with deep interest the article by Callaghan et al ${ }^{1}$ related to the efficacy of patellar brace in patients with patellofemoral (PF) osteoarthritis (OA) targeting knee pain and bone marrow lesions (BMLs). To our best knowledge, patellar brace in the management of PF disorders, including PF arthritis, was first introduced in $1981 .^{2}$ Since then only a few studies have examined the effects of patellar brace on the treatment of PF OA. This high-quality randomised controlled trial suggested that a PF brace reduced BML volume in the targeted compartment of the knee and relieved knee pain. These results were 'promising' in a situation where only a few conservative treatments have been consistently shown to affect structural findings. We really appreciate the work done by the authors. However, after reading the article, there are worthwhile issues that need to be explored.

A recent meta-analysis showed that there was a moderate statistically significant difference favouring the valgus brace group for improvement in pain and function compared with a control group that did not use an orthosis (blank control). ${ }^{3}$ However, compared with a control group that used a control orthosis (placebo), only a small improvement in pain, but not function, was observed. Although the authors fully explained the reasons why they did not choose a placebo as the control group, the possibility of overestimation of the effects cannot be ruled out. This should also be considered as a limitation in the present study. In addition, the authors did not mention the activity level of the included patients during the subsequent six weeks. Is there a possibility that patients with PF OA with patellar brace were more inclined to move less (such as knee-flexing exercises and weight-bearing activities)? Especially some knee-flexing activities, including stair climbing, arising from a chair and activities involving kneeling or squatting, can be hindered to some extent by the patellar brace. Thus, both knee pain and BMLs could be relieved temporarily. No doubt, we are very much looking forward to the results of a longer follow-up period. Furthermore, a previous meta-analysis concluded that placebo is effective in the treatment of OA, especially for pain, stiffness and self-reported function. ${ }^{4}$ In the absence of medium-term and long-term follow-up, is there a possibility that the effect of pain relief was just a placebo effect in the present study?

In addition to all the above, there are some other issues that need to be mentioned. First, the authors indicated that the severe knee was selected if both knees were eligible. We wonder whether the pain symptoms (yes or no) of the contralateral knee might have influenced the results. Second, a latest protocol of a randomised clinical trial assessing the effect of a knee brace on PF pain syndrome also did not choose a placebo control group. ${ }^{5}$ But the protocol made it clear that all the included patients (brace group and control group) will undergo supervised physiotherapy. In the present study, we wonder whether there was any standard care in each group. Third, the authors also did not mention the use of drugs, such as a painkiller, which potentially could affect the results. Fourth, the result of function improvement is worthy of expectation. Fifth, the authors indicated that potential participants had to be on stable medication for 3 months. Further explanation about these inclusion criteria would be better. Sixth, the authors missed to define $\mathrm{PF}$ in the first sentence of the abstract. Lastly, the green and red colours should be reversed in figure 1 .

We respect the great contributions of the authors and are looking forward to their response to these issues.

\section{Chao Zeng, Shu-guang Gao, Guang-hua Lei}

Department of Orthopaedics, Xiangya Hospital, Central South University, Changsha, Hunan Province, China

Correspondence to Professor Guang-hua Lei, Department of Orthopaedics, Xiangya Hospital, Central South University, Xiangya Road, Changsha, Hunan Province 410008, China; Igh9640@sina.cn

Contributors CZ: concept and writing; S-gG: writing; G-hL: concept and revising Competing interests None.

Provenance and peer review Not commissioned; internally peer reviewed.

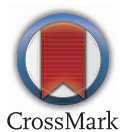

To cite Zeng C, Gao S-guang, Lei G-hua. Ann Rheum Dis 2015;74:e51.

Received 27 March 2015

Accepted 28 March 2015

Published Online First 9 April 2015

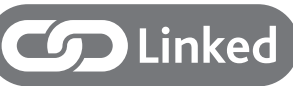

http://dx.doi.org/10.1136/annrheumdis-2015-207681

Ann Rheum Dis 2015;74:e51. doi:10.1136/annrheumdis-2015-207673

\section{REFERENCES}

1 Callaghan MJ, Parkes MJ, Hutchinson CE, et al. A randomised trial of a brace for patellofemoral osteoarthritis targeting knee pain and bone marrow lesions. Ann Rheum Dis 2015. Published Online First: 16 Jan 2015. doi:10.1136/annrheumdis2014-206376

2 Palumbo PM Jr. Dynamic patellar brace: a new orthosis in the management of patellofemoral disorders. A preliminary report. Am J Sports Med 1981;9:45-9.

3 Moyer RF, Birmingham TB, Bryant DM, et al. Valgus Bracing for knee osteoarthritis: a meta-analysis of randomized trials. Arthritis Care Res (Hoboken) 2015;67:493-501.

4 Zhang W, Robertson J, Jones AC, et al. The placebo effect and its determinants in osteoarthritis: meta-analysis of randomised controlled trials. Ann Rheum Dis 2008;67:1716-23.

5 Petersen W, Ellermann A, Rembitzki IV, et al. The Patella Pro study-effect of a knee brace on patellofemoral pain syndrome: design of a randomized clinical trial (DRKS-ID:DRKS00003291). BMC Musculoskelet Disord 2014. doi:10.1186/14712474-15-200 